\title{
Inclusion body myositis associated with Sjögren's syndrome
}

\author{
Maria Misterska-Skóra $\cdot$ Agata Sebastian • \\ Piotr Dzięgiel · Maciej Sebastian · Piotr Wiland
}

Received: 5 April 2012/ Accepted: 21 October 2012/Published online: 12 December 2012

(C) The Author(s) 2012. This article is published with open access at Springerlink.com

\begin{abstract}
Inclusion body myositis (IBM) belongs to the group of idiopathic inflammatory myopathies. It is a poorly understood disease, which affects skeletal muscles. IBM usually occurs as an isolated condition, but in some cases, it may be associated with another autoimmune disorder, Sjögren's syndrome. We report a case of a 47 -year-old woman with headaches, symptoms of trigeminal neuralgia, progressive weakness in muscles of the upper and lower extremities and symptoms of dry eyes and mouth. On admission, creatine kinase level was increased to 6,956 IU/ $\mathrm{mL}$ and lactate dehydrogenase (LDH) to $1,011 \mathrm{U} / \mathrm{L}$ in the serum. The increase in inflammatory factor (CRP, ESR) levels was not found. The diagnosis of inclusion body myositis associated with Sjögren's syndrome was established on the basis of clinical picture and diagnostic tests. In this therapy, methotrexate and methylprednisolone were administered. The considerable improved muscle strength in the upper and lower extremities, improved speech and swallowing, disappearance of headache and reduction in CPK and LDH levels were found 8 months after establishing the diagnosis. Treatment with methotrexate and methylprednisolone improved the clinical symptoms and quality of life of this patient and may offer a therapeutic
\end{abstract}

M. Misterska-Skóra · A. Sebastian ( $₫) \cdot$ P. Wiland Department of Rheumatology and Internal Medicine, Academic Hospital Wroclaw, Borowska Street 213, Wrocław, Poland e-mail: agatasebastian@vp.pl

P. Dzięgiel

Department of Histology and Embryology, Wroclaw Medical University, Chałubińskiego Street 6a, Wrocław, Poland

M. Sebastian

Department of Minimally Invasive Surgery and Proctology, Academic Hospital Wroclaw, Borowska Street 213, Wrocław, Poland option for some patients with IBM and concomitant Sjögren's syndrome.

Keywords Sjögren's syndrome · Inclusion body myositis · Therapeutic option - Quality of life

\section{Introduction}

Inclusion body myositis (IBM) is the most commonly acquired myopathy presenting over the age of 50, mostly in men. IBM belongs to the group of idiopathic inflammatory myopathies. It is a poorly understood disease, which affects skeletal muscles. The diagnosis of IBM should be suspected in an adult who has a disease with slow onset which involves distal and proximal muscles, especially foot extensors and deep flexor tendons. Facial muscles can be affected in IBM leading to the involvement of pharyngeal and neck flexor muscles, causing dysphagia and difficulty in holding up the head. In IBM, the autoimmune features coexist with degenerative features which include vacuolization and intrafiber deposition of amyloid and related molecules similar to those seen in Alzheimer's disease $[1,9]$.

The diagnosis is confirmed by the increase in serum muscle enzyme levels, electromyographic findings and muscle biopsy, with the presence of characteristic structures such as rimmed vacuoles and amyloid deposits. IBM usually occurs as an isolated condition, but in some cases, it may be associated with another autoimmune disorder, Sjögren's syndrome [2, 3]. Because of the rarity of the disease, lack of controlled trials and resistance to corticosteroids and other immunosuppressive therapies, the treatment for IBM is difficult and provides poor therapeutic benefits in comparison with the expected efficacy. 


\section{Case report}

We report a case of a 47-year-old woman, previously operated on for goiter, who was admitted to the Rheumatology and Internal Medicine Department at the Medical University in Wroclaw to diagnose the cause of muscle pain. The anamnesis revealed that for about 1 year, the patient had complained of recurrent headaches in the temporal and occipital region with the feeling of facial numbness, the symptoms of trigeminal neuralgia and progressive hearing disorders. These were the reasons for many neurological consultations and MRI of the head without finding a cause of the reported symptoms. On admittance to the ward, the patient complained of general weakness, lingering headache, increasing pain and weakness in muscles of the upper and lower extremities, inability to climb stairs, disorders of facial expressions, dysphagia and dysarthria, and weight loss due to lack of appetite (12 kg in 6 months). Furthermore, there had been symptoms of dry eyes and mouth for 3 months prior to the admittance.

Pain and weakness of proximal and distal muscles of the upper and lower extremities, heliotrope discoloration of the eyelids, increased tension in calf muscles, symmetrical Raynaud's phenomenon on the skin of fingers, and features of dry eyes and mouth were found on physical examination. Laboratory tests revealed high levels of creatine kinase (CK 6,956 IU/L, N: 145), lactate dehydrogenase (LDH 1,011 U/L, N: 247), and aminotransferases ALT (197 U/L, N: 0-35) and AST (335 U/L, N: 0-31). The following were also found: antinuclear antibodies (ANA) with a titer of 1: 3,200 in ELISA, high titer of antiTPO antibodies $(>1,000 \mathrm{IU} / \mathrm{mL}, \mathrm{N}: 0-5.61)$, the presence of rheumatoid factor $(15.6 \mathrm{IU} / \mathrm{mL}, \mathrm{N}$ : 0-14), mild hypergammaglobulinemia $(1.6 \mathrm{~g} / \mathrm{dL}, \mathrm{N}: 0.6-1.2)$, decreased serum level of complement factor $\mathrm{C} 4(0.09 \mathrm{~g} / \mathrm{L}, \mathrm{N}$ : 0.1-0.4) and hypercholesterolemia $(276 \mathrm{mg} / \mathrm{dL}, \quad \mathrm{N}$ : 130-200). The increase in inflammatory factor (CRP, ESR) levels was not found.

In MR angiography of the head, only non-specific changes in the subcortical white matter of the frontal lobes were visualized in the form of several small hyperintense foci on T2-weighted scans typical of vascular changes.

Because of the dry eyes and mouth symptoms, the salivary gland of the lip was excised and examined histopathologically. The histopathological picture revealed advanced inflammatory changes. The T score was 4 .

The EMG record of the biceps brachii muscle and gastrocnemius muscle revealed the primary muscle damage.

The musculocutaneous biopsy from gastrocnemius muscle was taken to establish the diagnosis of myopathy. The histopathological examination was suggestive of inflammatory myopathy which required further assessment

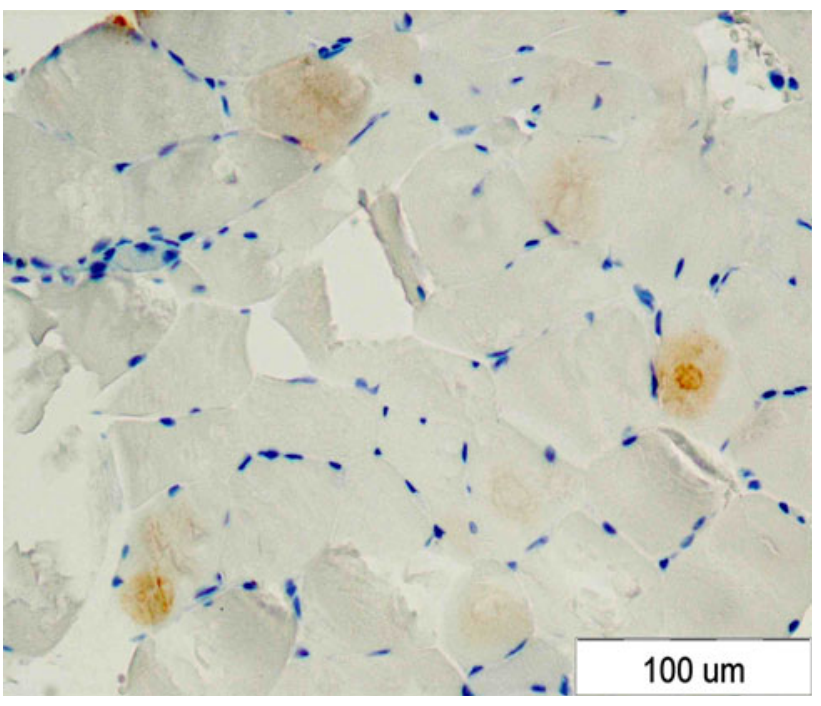

Fig. 1 Positive IHC reaction with anti- $\beta$ amyloid antibodies

using electron microscopy. Electron microscopic and immunohistochemistry examination of a muscle fragment confirmed the diagnosis of IBM (Figs. 1, 2).

The diagnosis of inclusion body myositis associated with Sjögren's syndrome was established on the basis of clinical picture and diagnostic tests. The patient fulfilled the Griggs et al. criteria for IBM and the EEC consensus criteria for Sjögren's syndrome. Methotrexate once weekly at a dose of $15 \mathrm{mg}$ and methylprednisolone at a daily dose of $16 \mathrm{mg}$ were administered, which resulted in gradual clinical improvement and reduction in muscle enzyme levels.

The muscle strength improved considerably in the upper and lower extremities; improved speech and swallowing, disappearance of headache and slow reduction in CPK level to $340 \mathrm{IU} / \mathrm{L}$ and $\mathrm{LDH}$ to $256 \mathrm{U} / \mathrm{L}$ were found 8 months after establishing the diagnosis.

\section{Discussion}

Muscle involvement is one of the clinical features of Sjögren's syndrome. The frequency of muscle changes is estimated at $3 \%$ [3]. IBM, dermatomyositis and polymyositis belong to the forms of immune-mediated myositis [4]. In most cases, the symptoms of dryness precede the symptoms of IBM. Myositis may be associated with extraglandular symptoms such as arthralgias/arthritis, peripheral neuropathy, interstitial kidney disease, primary biliary cirrhosis, cutaneous changes and hepatomegaly. Muscle involvement appears to be symmetrical [2,4].

The association between IBM and SS has not been found yet. There is a strong association between IBM and the class II major histocompatibility allele HLA-DR3 


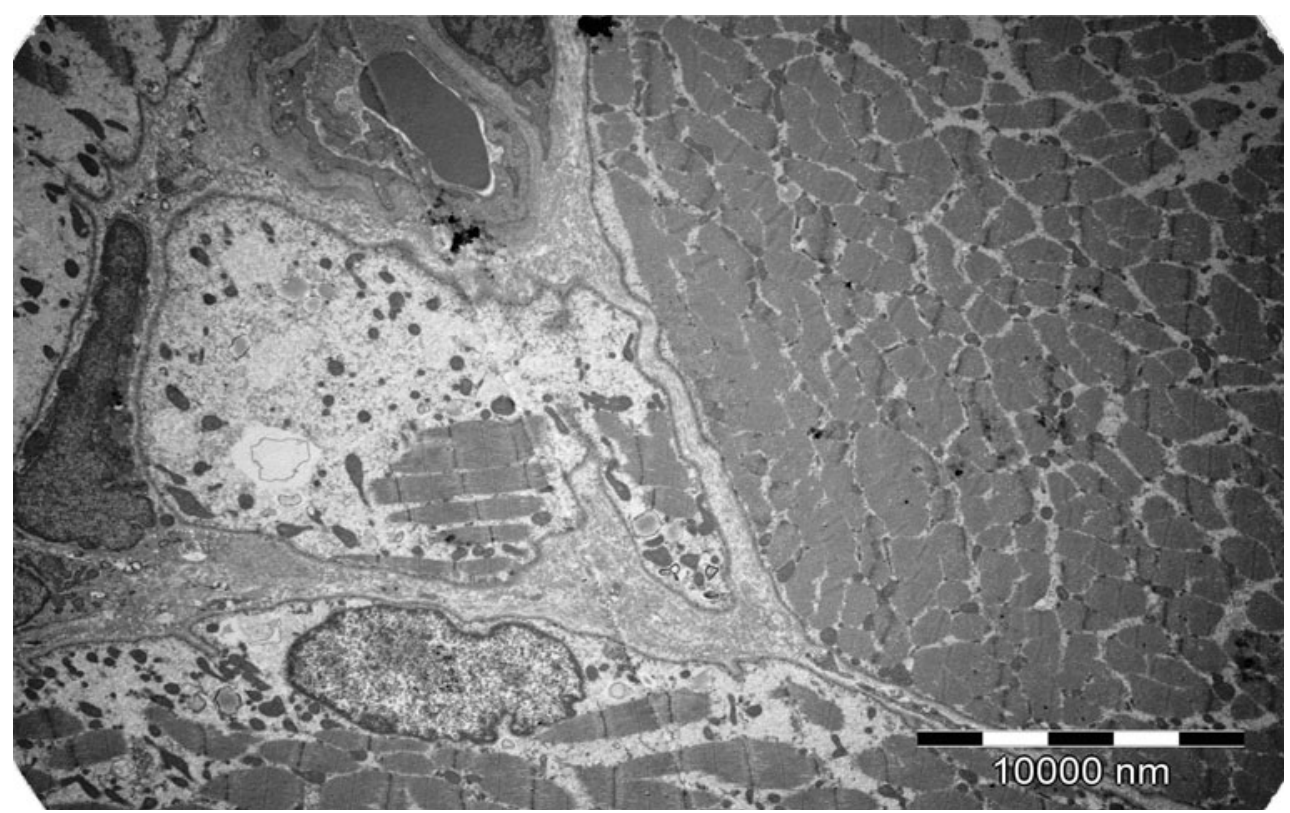

Fig. 2 Special features of IBM in electron microscopic examination

$[5,6]$. There is a major genetic association with allelic markers of the 8.1 MHC ancestral haplotype (HLA-A1, B8, DR3) [6]. In normal muscle fibers, MHC class I or II antigens are not detected. In IBM, MHC expression is the early event that can be detected in this part of muscles with inflammation [7]. It is not clear which aspect, either degenerative or inflammatory, is the primary component in the IBM pathogenesis $[8,9]$. Degeneration in muscle leads to the muscle fiber atrophy and fiber apoptosis and causes muscle weakness. In IBM, protein depositions include $\beta$ amyloid and related molecules similar to those seen in Alzheimer's disease, suggesting that similar mechanisms lead to tau phosphorylation in both diseases [9]. The proteins can be found inside inclusion or free within the cytoplasma [7]. Currently, there is no one specific molecule that could serve as a unique biomarker for IBM.

PSS induces polyclonal B lymphocyte activation, leading to the production of antibodies that react with antinuclear antibodies (ANA) and soluble nuclear antigens SS-A (Ro) and SS-B (La). The inflammatory infiltrates in salivary glands consist mainly of CD4+ $\mathrm{T}$ lymphocytes and, to a considerably smaller extent, $\mathrm{CD} 8+\mathrm{T}$ and $\mathrm{B}$ lymphocytes $[10,11]$. The muscle infiltrates in IBM, similar to the Sjögren's syndrome, consist of a mixture of B and T cells. The proportions of $\mathrm{CD} 8+$ and $\mathrm{CD} 4+$ cells are various according to the case. Sometimes CD8+ cells are dominant and sometimes CD4+ cells [4]. On the other hand, in both diseases, autoimmune pathogenesis mediated in either case by cytotoxic $\mathrm{T}$ cells is present [11]. A common genetic predisposition is linked with MHC in IBM and SS [4].
One of the characteristic symptoms of IBM is dysphagia [12], which Cox et al. found in $65 \%$ of patients with IBM. Dysfunction of the cricopharyngeal sphincter was found in $37 \%$ of patients. Dysphagia during the course of disease is probably caused by the inflammatory involvement of the cricopharyngeal muscles. Facial muscles may be affected in IBM similar to the pharyngeal and neck flexor muscles, causing dysphagia and difficulties holding up the head [7].

Most patients with IBM are unresponsive to treatment with glucocorticoids and conventional immunosuppressive therapy. Current recommendations for the treatment for IBM do not exist. Hydroxychloroquine, azathioprine, methotrexate and methylprednisolone were used, but with poor clinical response and constant, slow deterioration of muscle function $[2,13]$. The use of immunoglobulins was connected with improvement in $25 \%$ of patients. This effect was short. IBM has primarily an autoimmune pathogenesis, mediated by cytotoxic $\mathrm{T}$ cells [7]. A promising therapy may be the use of alemtuzumab-a $\mathrm{T}$ cell-depleting monoclonal antibody. It was used in a small uncontrolled study and showed that the depletion of peripheral $\mathrm{T}$ cells also caused the reduction in T cells in the muscle and may stabilize the illness [14]. Physiotherapy is recommended in all the cases.

The evident clinical improvement in the reported case resulted probably from a good response to treatment for concomitant Sjögren's syndrome.

Nowadays, early and correct diagnosis of IBM appears to be only a treatment challenge because the current therapy is the treatment of choice. 
Open Access This article is distributed under the terms of the Creative Commons Attribution License which permits any use, distribution, and reproduction in any medium, provided the original author(s) and the source are credited.

\section{References}

1. Dalakas MC (2006) Sporadic inclusion body myositis-diagnosis, pathogenesis and therapeutic strategies. Nat Clin Pract Neurol 2:437-447

2. Kanellopoulos P, Baltoyiannis C, Tzioufas AG (2002) Primary Sjögren's syndrome associated with inclusion body myositis. Rheumatol 41(4):440-444

3. Kraus A, Cifuentes M, Villa AR et al (1994) Myositis in primary Sjogren's syndrome. Report of 3 cases. J Rheumatol 21:649-653

4. Rojana-udomsart A, Needham M, Luo YB et al (2004) Associations with autoimmune disorders and HLA class I and II antigens in inclusion body myositis. Clin Neurol Neurosurg 63(12):2396-2398

5. Garlepp MJ, Laing B, Zilko PJ et al (1994) The association of sporadic inclusion body myositis and Sjögren's syndrome in carriers of HLA-DR3 and the 8.1 MHC ancestral haplotype. Clin Neurol Neurosurg 113(7):559-563

6. Dalakas MC (2011) Review: an update on inflammatory and autoimmune myopathies. Neuropathol Appl Neurobiol 37(3):226-242
7. Ghirardello A, Zampieri S, Tarricone E et al (2011) Cutting edge issues in polymyositis. Clin Rev Allergy Immunol 41(2):179-189

8. Askanas V, Engel WK, Nogalska A (2009) Inclusion body myositis: a degenerative muscle disease associated with intramuscle fiber multi-protein aggregates, proteasome inhibition, endoplasmic reticulum stress and decreased lysosomal degradation. Brain Pathol 19(3):493-506

9. Nogalska A, D'Agostino C, Engel WK et al (2011) Novel demonstration of conformationally modified tau in sporadic inclusionbody myositis muscle fibers. Neurosci Lett 503(3):229-233

10. Fauchais AL, Martel C, Gondran G et al (2010) Immunological profile in primary Sjögren syndrome: clinical significance, prognosis and long-term evolution to other auto-immune disease. Autoimmun Rev 9(9):595-599

11. Dlakas MC (2010) Inflammatory muscle diseases: a critical review on pathogenesis and therapies. Curr Opin Pharmacol 10:346-352

12. Cox FM, Verschuuren JJ, Verbist BM et al (2009) Detecting dysphagia in inclusion body myositis. J Neurol 256(12): 2009-2013

13. Dalakas MC (2011) Inflammatory myopathies: management of steroid resistance. Curr Opin Neurol 24(25):457-462

14. Dalakas MC, Rakocevic G, Schmidt J et al (2009) Effect of Alemtuzumab (CAMPATH 1-H) in patients with inclusion-body myositis. Brain 132(Pt 6):1536-1544 